\title{
Microstructural analysis of human white matter architecture using polarized light imaging: views from neuroanatomy
}

\author{
Hubertus Axer ${ }^{1}{ }^{*}$, Sindy Beck ${ }^{1}$, Markus Axer ${ }^{2}$, Friederike Schuchardt ${ }^{1}$, Jörn Heepe ${ }^{1}$, Anja Flücken ${ }^{1}$, \\ Martina Axer ${ }^{3}$, Andreas Prescher ${ }^{4}$ and Otto W. Witte ${ }^{1}$
}

\author{
${ }^{1}$ Hans Berger Department of Neurology, Jena University Hospital, Jena, Germany \\ ${ }_{2}^{2}$ Institute of Neuroscience and Medicine, Research Center Jülich, Jülich, Germany \\ ${ }^{3}$ Department of Psychiatry and Psychotherapy, Friedrich-Schiller-University, Jena, Germany \\ ${ }^{4}$ Institute of Anatomy, Technical University Aachen, Aachen, Germany
}

\section{Edited by:}

Claus Hilgetag, Jacobs University Bremen, Germany

Reviewed by:

Basilis Zikopoulos, Boston University, USA

Marc Tittgemeyer,

Max-Planck-Institute for Neurological

Research, Germany

*Correspondence:

Hubertus Axer, Hans Berger

Department of Neurology, Jena

University Hospital,

Friedrich-Schiller-University Jena,

Erlanger Allee 101, D-07747 Jena,

Germany.

e-mail: hubertus.axer@med.

uni-jena.de
To date, there are several methods for mapping connectivity, ranging from the macroscopic to molecular scales. However, it is difficult to integrate this multiply-scaled data into one concept. Polarized light imaging (PLI) is a method to quantify fiber orientation in gross histological brain sections based on the birefringent properties of the myelin sheaths. The method is capable of imaging fiber orientation of larger-scale architectural patterns with higher detail than diffusion MRI of the human brain. PLI analyses light transmission through a gross histological section of a human brain under rotation of a polarization filter combination. Estimates of the angle of fiber direction and the angle of fiber inclination are automatically calculated at every point of the imaged section. Multiple sections can be assembled into a 3D volume. We describe the principles of PLI and present several studies of fiber anatomy as a synopsis of PLI: six brainstems were serially sectioned, imaged with PLI, and 3D reconstructed. Pyramidal tract and lemniscus medialis were segmented in the PLI datasets. PLI data from the internal capsule was related to results from confocal laser scanning microscopy, which is a method of smaller scale fiber anatomy. PLI fiber architecture of the extreme capsule was compared to macroscopical dissection, which represents a method of larger-scale anatomy. The microstructure of the anterior human cingulum bundle was analyzed in serial sections of six human brains. PLI can generate highly resolved 3D datasets of fiber orientation of the human brain and has high comparability to diffusion MR. To get additional information regarding axon structure and density, $\mathrm{PLI}$ can also be combined with classical histological stains. It brings the directional aspects of diffusion MRI into the range of histology and may represent a promising tool to close the gap between larger-scale diffusion orientation and microstructural histological analysis of connectivity.

Keywords: polarized light imaging, brainstem, pyramidal tract, internal capsule, cingulum, extreme capsule

\section{INTRODUCTION}

Of late, the discussion relating to exploring the human connectome to attain a comprehensive structural description of the overall connectivity in the human brain has gained increasing attention (Sporns et al., 2005; Sporns, 2011). Mapping the anatomical fiber pathways connecting the various regions of the human brain is the basis for comprehending its complex function. In this context the advance of MRI methods for mapping the human connectome has recently been discussed (Hagmann et al., 2010). The method is non-invasive and allows the in vivo study of the human brain with regard to anatomical connectivity (diffusion MRI) as well as functional interrelationships [functional neuroimaging, e.g., functional magnetic resonance imaging (fMRI), but also PET, etc.]. Although, several methods for mapping anatomical connectivity extending from the macroscopic to molecular scale levels are established it is difficult to integrate these multiply-scaled data into a single concept. This difficulty arises since different imaging methods use different coordinate systems. The assembly of microscopical slices into a $3 \mathrm{D}$ dataset is possible but the projection of these data into a 3D reference coordinate system of the human brain is not generally done. There is a need for a reference coordinate system which is applicable to a wide range of different imaging modalities. Moreover, each method only shows a selective view on the object, such as connectivity, nerve fiber architecture at a specific location in the brain, diameter of fibers, fiber density, as well as fiber orientation, and many more. The method used depends of the hypothesis to be proven. A further difficulty arises from the fact that the large living human brain generally cannot be studied using different methods in parallel and diffusion MRI and fMRI is not possible to be done or at least is hindered in the formalin fixed cadaver brain. 
To talk about scales and structure it is indispensable to consider the dimensions of the anatomical structures to be imaged. A nerve fiber is composed of the axon plus its myelin sheath. As viewed under the electron microscope, the size of myelinated fibers in the human corpus callosum range from 0.2 to more than $10 \mu \mathrm{m}$ in diameter, whilst the diameters of unmyelinated fibers span 0.1$1 \mu \mathrm{m}$ (Aboitiz et al., 1992). Fiber density in the corpus callosum is between 300,000 and 400,000 per $\mathrm{mm}^{2}$. The number of fibers in the corpus callosum is in the order of $10^{8}$, whereas the number of cortico-cortical projections in one hemisphere is at least one magnitude higher (Schüz and Preißl, 1996). In the human pyramidal tract, $87.9 \%$ of fibers are below $4 \mu \mathrm{m}, 10.7 \%$ range from 4 to $10 \mu \mathrm{m}$, and $1.4 \%$ of fibers are larger than $10 \mu \mathrm{m}$ (Graf von Keyserlingk and Schramm, 1984). Fiber density in the pyramid of the medulla oblongata is about 11,000 fibers per $\mathrm{mm}^{2}$.

Single nerve fibers are mostly collected in fiber bundles. For example, in the anterior limb of the internal capsule, the fiber bundles of the frontopontine tract are arranged in sheaths with a diameter of about $100-150 \mu \mathrm{m}$ that intermingle with fibers from the anterior thalamic peduncle (Axer et al., 1999a). At this scale the detection of a single axon may not be critical. On the contrary, a lower resolution might be better suited to visualize the structure of larger fiber bundles. Classical fiber tracts, e.g., the pyramidal tract, or fasciculi, e.g., the arcuate fasciculus, are collections of fibers which have been anatomically defined mainly inspired from macroscopical dissection (Ludwig and Klingler, 1956). Terms like "pathway" or "stream" are not anatomical descriptions but are mainly used in the context of functional considerations of connectivity.

In the CNS, compact fiber bundles such as the pyramidal tracts at the level of the medulla oblongata are the exception. Generally, different fiber bundles intermingle with each other in the white matter. A very critical region is the subcortical white matter directly adjacent to the gray matter, where single nerve fibers intermingle diffusely. Nerve fibers are generally not arranged in bundles at this location and these regions are therefore difficult to be imaged with diffusion MRI, due to its limited resolution power.

Obviously, the resolution of the imaging system strongly influences the level of architectural scale to be visualized. In Figure 1, examples of the scale of various anatomical structures together with the resolution of different imaging modalities can be seen. For instance, electron microscopy, confocal laser scanning microscopy (CLSM), and classical light microscopy are used for visualization of every single axon, while MR will focus on larger architectural pattern, e.g., larger fiber tracts. Ex vivo MRI in mice (9.4 T) achieves a resolution in the range of $100 \mu \mathrm{m}$ or less (Zhang et al., 2011), while in vivo diffusion MRI of the whole human brain will maximally reach a resolution of $0.5 \mathrm{~mm}$ (Finsterbusch, 2009). Diffusion MR allows the inference of $3 \mathrm{D}$ fiber orientation data in the human brain and, therefore, enables the tracking of fiber pathways in the brain based on various methods such as streamline tractography, probabilistic fiber tracking, and several others (Hagmann et al., 2010). Overall, the relatively low magnification of in vivo diffusion MRI of the human brain solely allows the analysis of larger-scale architectural patterns.

In contrast, using high resolution anatomical methods, e.g., electron microscopy, it is possible to visualize single axons,

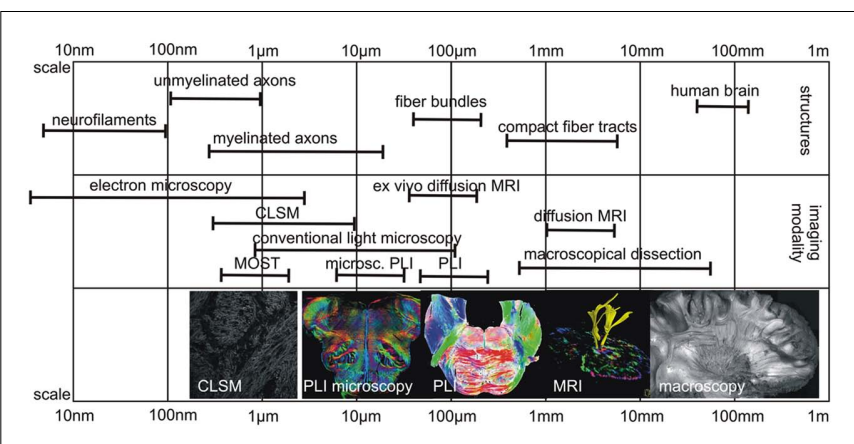

FIGURE 1 | Scales of anatomical structure and image modality. The image shows the different scales of anatomical structures of interest and the magnification of different image modalities, which can be used to study white matter architecture. Abbreviations: CLSM, confocal laser scanning microscopy; MOST, micro-optical sectioning tomography (Li et al., 2010).

dendrites, dendritic spines, and synapses at the highest resolution (nm-range), even in 3D (Briggman and Denk, 2006). The recently described micro-optical sectioning tomography (MOST) is able to collect highly resolved 3D data of a complete Golgi-stained mouse brain with a voxel size of $0.33 \mu \mathrm{m} \times 0.33 \mu \mathrm{m} \times 1 \mu \mathrm{m}$ (Li et al., $2010)$. Such methods, however, only cater for visualization of volumes that are smaller in size than the human brain due to the enormous amount of data they accumulate. In future, high performance computing might provide analysis tools and procedures to study specimen such as the human brain reasonably. Difficulties arise, therefore, in the transfer from human connectivity data from high-scaled anatomical investigations. The higher the magnification of the method is, the smaller is the field of view or the volume of view. Thus, there is a gap between analyzing methods of the human brain and highly resolved anatomical methods.

Polarized light imaging (PLI), a method based on the birefringent properties of the myelin sheaths, is used to quantify fiber orientation in gross histological brain sections. We developed PLI more than a decade ago during the late 1990s (Axer et al., 2000, 2001). The idea was inspired from studies of electrical impedance measurements using impedance measurements in the human brain as an online verification of the position of the electrode during stereotaxic procedures. Impedance in the white matter is mainly influenced by the orientation of the nerve fibers in relation to the electrode (Axer et al., 1999b). Hence, an anatomical method was needed to construct a highly detailed map of the fiber orientation in the human brain in order to create a $3 \mathrm{D}$ atlas of fiber orientation as an anatomical correlate of impedance measurements (Axer et al., 2002a). Although the impedance principle for white matter was not followed up, the introduction of diffusion imaging to visualize fiber orientation in the living human brain has led to a growing interest in connectivity studies of the human brain (Catani et al., 2002; Jones, 2011).

This paper aims to describe PLI for imaging the human brain to investigate anatomical connectivity under the aspect of macroscopic and microscopic scales together with its relation to other methods. Herein, we present four different circumscript PLI studies, which show the relation of this new method to other imaging procedures such as diffusion tensor imaging (DTI), macroscopical 
dissection, and CLSM. The following four studies have not been designed to determine the problem of scale in connectivity, rather each study demonstrates specific anatomical aspects relevant for PLI with respect to other imaging apertures and also enables the interpretation of the results in the context of functionality of fiber tracts:

1. Study of the cingulum bundle, a fiber bundle mainly involved in performing complex cognitive and neuropsychological tasks, is an example of the use of PLI and macroscopical dissection to obtain an anatomical description of an association fiber tract in the human brain.

2. Polarized light imaging analysis of the human brainstem shows the use of PLI in imaging compact fiber tracts in this study. PLI is applied for detection and segmentation as well as for morphometric analysis of these fiber tracts. Although, the analysis of the pyramidal tract is also undertaken with diffusion MRI such as for Wallerian degeneration after stroke (Grässel et al., 2010), smaller fiber tracts such as the lemniscus medialis and many others are difficult to be reliably imaged using MRI.

3. The internal capsule is a structure of white matter composed of diverse fiber bundles of varying orientation and differing dimensions. Thus, the internal capsule is a bottleneck of fibers trafficking signals from the cortex to the thalamus and the brainstem, and vice versa. Since it represents a clinically essential and complex structure, an imaging system with a larger magnification, such as PLI may be of benefit for investigating the internal capsule. Therefore, the use of CLSM is used for comparison to analyze very highly resolved nerve fiber architecture at different points of the internal capsule.

4. The extreme capsule constitutes a collection of diversely associated fiber pathways located between the claustrum and the insular cortex. Recently, interest has been sparked in the structure as fMRI and DTI studies revealed a functional involvement of these fiber pathways in language tasks (Saur et al., 2008). Thus, PLI was used to analyze fiber orientation in relation to macroscopical dissection of this brain region.

\section{MATERIALS AND METHODS PRINCIPLES OF PLI}

Currently polarized light has a broad range of technological applications that cover diverse industrial and scientific fields, including optical communication, display technology, solid state physics, and biomedical optics (Brosseau, 1998). In many of these applications, polarized light is used to visualize the birefringence and, consequently, to demonstrate the spatial structure of the material under study. Generally speaking, a material is referred to as being birefringent if its molecular structure has anisotropic properties, i.e., when shape and orientation of individual molecules introduce directional dependencies. Such directional dependencies become detectable when linearly polarized light passes through a sample. For anisotropic media, the velocity of light $v$ will vary depending on the direction of the advancing light wave and its state of polarization. This observation can be described by an elliptically shaped oblate surface, the refractive index ellipsoid or the indicatrix, characterizing the structural composition of the material by means of direction-dependent refractive indices $n$ ( $=$ the ratio of light velocities in vacuum and in the material). The indicatrix is defined by three principal refractive indices along the principal axes of the medium. If two of these principal refractive indices are equal due to symmetry reasons, the medium is said to be uniaxial.

It has been well known for some time that the white matter of the brain has a distinct anisotropy and, thus, exhibits optical birefringence (Brodmann, 1903; Göthlin, 1913; Schmidt, 1924; Schmitt and Bear, 1935; Kretschmann, 1967; Wolman, 1970, 1975; Miklossy and Van der Loos, 1991). White matter basically consists of closely packed myelinated fibers. The myelin sheaths are multilayer membranes that are wrapped around the axons (Martenson, 1992). About 70-85\% of the myelin sheaths are composed of radially ordered lipids (cholesterol, sphingolipids, and phospholipids) whilst only $15-30 \%$ consist of proteins [proteolipidprotein (PLP) and myelin basic protein (MBP)] arranged tangentially to the axon. Vidal et al. (1979) were able to attribute the birefringence of the white matter mainly to the radially arranged lipids of the myelin sheaths and observed an overall negative uniaxial birefringence. As a consequence, a myelinated fiber can be described by two principal refractive indices, parallel $\left(n_{\mathrm{ff}}\right)$ and orthogonal $\left(n_{\mathrm{cc}}\right)$ to the physical fiber axis. Given that the difference of $n_{\mathrm{cc}}$ and $n_{\mathrm{ff}}$ is small and the fiber is inclined by the angle $\alpha$ with respect to the front of the advancing light wave (Scheuner and Hutschenreuter, 1972; Larsen et al., 2007), the optical birefringence $\Delta n$ of the fiber can be approximately expressed by:

$\Delta n \approx\left(n_{\mathrm{cc}}-n_{\mathrm{ff}}\right) \cos ^{2} \alpha$.

A beam of linearly polarized light will, on passing through a thin section of white matter, split up into two perpendicular components, the ordinary and the extraordinary ray. Due to different propagation velocities of the two rays, the beam in general becomes elliptically polarized with a phase shift $\delta$ and a difference in amplitude depending on the local fiber orientation. If the light travels along the fiber axes, the light polarization will remain unaltered. The induced phase shift is a function of the wavelength of the light source $\lambda$, the section thickness $d$, and the optical birefringence $\Delta n$ :

$\delta=2 \pi d \Delta n / \lambda$

An apparatus that enables the measurement of the described birefringence effects is referred to as polarimeter. The simplest setup of a polarimeter consists of two crossed linear polarizers with an object stage in between, a CCD camera, and a light source. The polarizers are rotatable (by discrete angles $\rho$ ) around the fixed sample. Since the optical system is exclusively composed of linear optical elements and the light beam that enters the sample can be considered to be fully polarized after passing the first polarizer, the application of Jones calculus (Jones, 1941) is permitted to describe the light transmittance $I$ through the polarimeter:

$I=I_{0} \sin ^{2}(2 \rho-2 \varphi) \sin ^{2}(\delta / 2)$.

$I_{0}$ describes the intensity of the incident light, $\rho$ is the azimuth of the transmission axis of the first linear polarizer, $\varphi$ is the projection of the fiber into the polarizer's plane relative to the null position of the polarimeter $\left(\rho=0^{\circ}\right)$, and $\delta$ is the phase shift as specified in Eqs 1 and 2. 
A more advanced polarimeter system houses an additional rotating quarter-wave retarder above the object stage, which leads to the following formula for the light transmittance $I$ :

$I=I_{0} / 2(1+\sin (2 \rho-2 \varphi) \sin (\delta))$.

In conclusion, these types of polarimeter systems sense both the fiber inclination $\alpha$ and the fiber direction $\varphi$. Therefore, by measuring the transmitted light intensity per image pixel at discrete rotation angles $\rho$ and subsequent data analysis, the system provides direct access to the locally prevailing 3D fiber orientations. This imaging procedure is known as PLI.

\section{PLI SYSTEM AND PREPARATION OF THE SAMPLES}

The polarimeter system used herein (cf. Larsen et al., 2007) consists of a filter combination of a pair of horizontally mounted crossed polars and a removable quarter-wave plate (B\&W Filter, Schneider, Bad Kreuznach, Germany). Diffuse light of five fluorescent tubes behind a diffusing screen (Osram $\mathrm{GmbH}$, München, Germany, Universal White) is passed through the system from below. The transmitted light is imaged by a downward pointing CCD camera (Axiocam HR, Carl Zeiss, Göttingen, Germany, basic resolution of $1388 \times 1040$ pixel). This system allows the filters to be rotated while maintaining their relative orientation constant, and keeping the sample fixed on a stage. The images were acquired using the AxioVision software (Carl Zeiss, Göttingen, Germany). The magnification of the system was such as to result in pixels of dimension $64 \mu \mathrm{m} \times 64 \mu \mathrm{m}$ over a thickness of $100 \mu \mathrm{m}$.

For each brain section, nine images separated by $10^{\circ}$ rotations of the filters using the crossed polars only and nine images separated by $20^{\circ}$ rotations of the filters using an introduced quarter-wave plate were acquired (Figure 2). Sinusoids were fitted to the two sequences of nine intensity values at each pixel to recover direction $\varphi$ (in-plane orientation) and inclination $\alpha$ (out-of-plane orientation) information by means of Eqs 3 and 4 . The relationship between inclination and maximum intensity of transmitted light is not linear (cf. Eqs 1-4), and it is clear that the accuracy with which the inclination can be estimated will be best for inclinations between $30^{\circ}$ and $60^{\circ}$.

Sections of human cadaver brains fixed in $4 \%$ aqueous formalin solution for at least 3 weeks were used for PLI. The brains were taken from persons (without history of neurologic or psychiatric disease) who donated their body for anatomical study before death. All brains were collected from the body donor program of the Institute of Anatomy at the Technical University Aachen (RWTH). After cryoprotection, brain slabs of the region of interest were serially sectioned with a cryostat microtome (CM3050 S, Leica Microsystems, Bensheim, Germany) at a thickness of $100 \mu \mathrm{m}$. The sections were mounted with the aqueous mounting medium Aquatex ${ }^{\mathrm{TM}}$ (Merck, Darmstadt, Germany) and coverslipped without staining.

Image processing was realized with scripts written in MATLAB 7.7.0 (MathWorks Inc., Natick, MA, USA) including the Image Processing Toolbox. The method used for 3D reconstruction has been described elsewhere in detail (Axer et al., 2002b) In short, rigid (isomorphic) transformations were computed on the serial sections of the brain. Maximum intensity maps (which represent

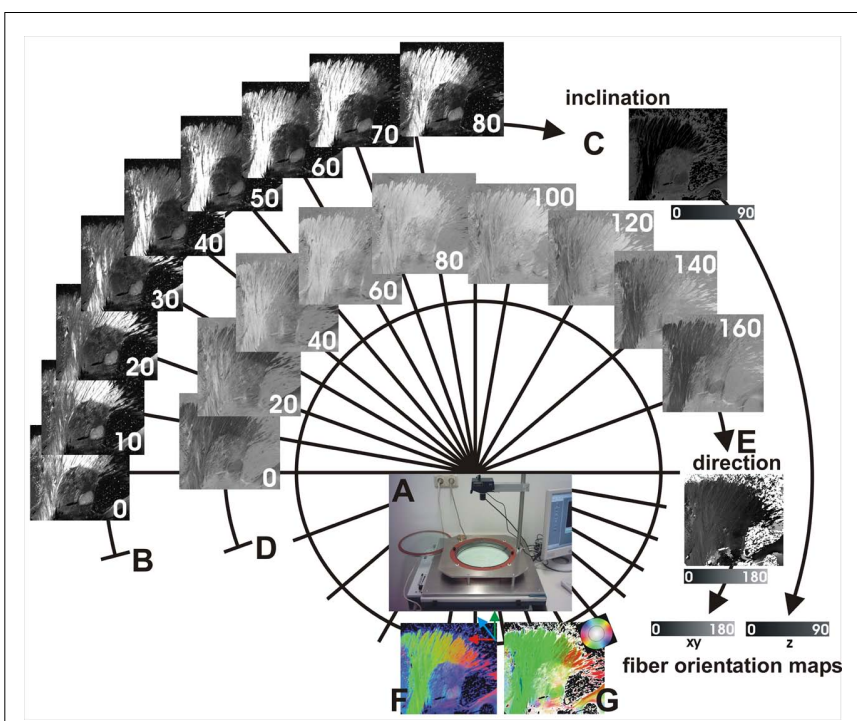

FIGURE 2 | Image acquisition and image processing of PLI. Equipment of the PLI system (A) consists of two rotatable perpendicularly oriented polarization filters and an insertable quarter-wave plate. For each section, nine images separated by $10^{\circ}$ rotations of the filters using two crossed polars are acquired (B), which are used to calculate angles of fiber inclination (C). In addition, nine images separated by $20^{\circ}$ rotations of the filters using an additionally introduced quarter-wave plate (D) are used to calculate angles of direction (E). Angles of inclination and angles of direction in every pixel of the section define a vector representing the major $3 \mathrm{D}$ fiber orientation at that point. Fiber orientation maps (FOMs) can be visualized using different color schemes $\mathbf{( F , G )}$

the brightest intensity of transmitted light in each pixel derived from each polarization sequence using the crossed polars only) were used for this purpose because of their good anatomical contrast. Each image was translated and rotated with respect to its predecessor. In case of rotations, the angles of direction have to be adjusted according to the degree of rotation. The minimized Euclidean distance was used as criterion for the best fit between the succeeding slices (Axer et al., 2002b).

Three dimensional Slicer 2.6 (www.slicer.org) was used for visualization of the 3D datasets and for segmentation of fiber tracts. It is an open-source, cross-platform application for visualizing and analyzing medical image data (Gering et al., 2001). SPSS 11.5.1 (SPSS Inc., Chicago, IL, USA) was used for statistical analysis.

\section{RESULTS}

\section{CINGULUM BUNDLE}

Six formalin fixed human cadaver brains were macroscopically dissected (four males, two females, median 79 years, range $72-$ 88 years). A $1.5-\mathrm{cm}$ thick slabs of the mediofrontal brain including the anterior cingulum bundle were cut in four separate pieces, serially sliced and analyzed using PLI. The target of this analysis was a parcellation of the anterior cingulum bundle according to fiber orientation (Figure 3). The anterior part of the cingulum is defined as the fiber bundles located above the corpus callosum and running into the frontal lobe.

The anterior cingulum bundle can topographically be subdivided into a supracallosal, pregenual, and subgenual part for 


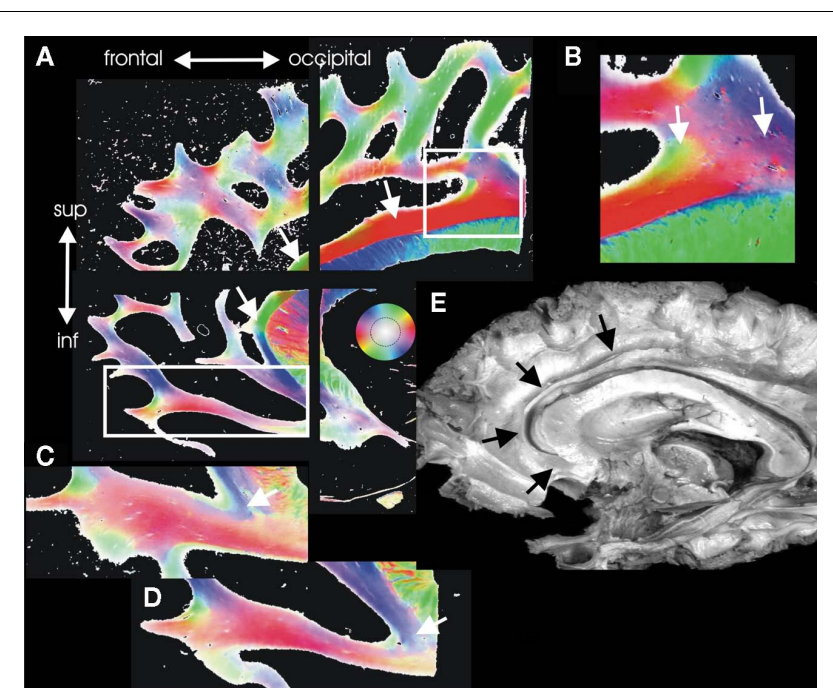

FIGURE 3 | Architectural parcellation of the anterior cingulum bundle. The cingulum bundle is marked by the white arrows in the Fiber Orientation Maps [FOMs (A)]. (E) Shows the cingulum bundle in a dissected human brain for comparison (black arrows). The anterior supracallosal and the pregenual part of the cingulum bundle is a single compact fiber bundle [white arrows in (A)]. In its midcingulate posterior part, the supracallosal cingulum bundle gets several inputs from fiber bundles coming from the adjacent white matter. This part is magnified in (B) and shows intermingling fiber bundles running into the cingulum bundle visualized in green and blue color (arrows). In the subgenual part, the fibers of the cingulum bundle curve and spread into the orbitofrontal regions [(C,D) arrows].

purposes of systematic anatomical description. The anterior part of the supracallosal cingulum bundle is a single compact fiber bundle without intermingling fiber bundles and without significant inputs. In its posterior part (midcingulate), the supracallosal cingulum bundle receives several inputs from fiber bundles originating from the adjacent white matter. Therefore, the midcingular segment gets inputs from fiber bundles oriented to motor and premotor cortices and parietal cortex.

In the pregenual part, the cingulum bundle is rather compact and curves around the genu of the corpus callosum. The fibers of the cingulum bundle in the subgenual part curve and spread into the orbitofrontal white matter. These fibers are diffusely intermingled with fibers deriving from the medial forebrain bundle and the forceps minor of the corpus callosum. A part of the cingulum further proceeds in the direction of the limen insulae.

\section{HUMAN BRAINSTEM}

Six human brainstems (three males and three female, median 81.5 years, range 65-92 years) were serially sectioned axially to the brainstem axis and every second slice was imaged using PLI. Figure 4 shows sections through the medulla oblongata, pons, and mesencephalon. The major fiber tracts in the brainstem could be identified by means of PLI data, i.e., pyramidal tracts, lemniscus medialis, medial longitudinal fascicle, cerebellar peduncles, and others. Although PLI data of the larger fiber tracts show similarities to DTI results (Figures 4A,B), the advantage of PLI lies in the greater resolution which additionally allows for localization of smaller fiber bundles.
The serial fiber orientation maps were registered threedimensionally and the volumes were imported into the Slicer software. Pyramidal tract and lemniscus medialis were manually segmented according to the fiber orientation maps (Figure 5). The volumes of these fiber tracts were estimated and related to the volume of the whole white matter of the brainstem in order to normalize according to individual brain size. Figure 6A shows the normalized measurements of the six brains.

The volumes of the pyramidal tracts showed a negative correlation to age (Figure 6B, left pyramidal tracts: Pearson correlation coefficient $-0.930, p=0.007$, right pyramidal tracts: Pearson correlation coefficient $-0.882, p=0.02$ ). In contrast, no such relationship could be found for the volumes of the medial lemniscus (Figure 6C) or the volume of the whole brainstem white matter.

\section{INTERNAL CAPSULE}

The internal capsule is a collection of intermingling fiber bundles of different fiber systems, i.e., the pyramidal tract, thalamic radiations, corticopontine fiber systems, and others. Using CLSM, it was demonstrated that the individual orientation of distinct fiber bundles could be used to describe a parcellation of the internal capsule (Axer and Keyserlingk, 2000). Right-left comparison of morphometric parameters of these bundles demonstrated that more and smaller bundles of the frontopontine fiber tract were located on the left-hand side than on the right-hand side of the anterior limb of the internal capsule (Axer et al., 1999a).

Confocal laser scanning microscopy allows information to be collected from well-defined optical sections through a fluorescent sample. This is done through sequential illumination focusing on one volume element of the specimen at a time (Wright et al., 1993). Stacks of serial optical sections allow a 3D reconstruction of the fiber architecture and provide good quality information regarding the orientation of the fibers at a high resolution. The myelin sheaths of the nerve fibers can be labeled with the fluorescent dye DiI and has been used to analyze fiber architecture in the internal capsule systematically (Axer and Keyserlingk, 2000).

At the level of the internal capsule, the different tracts do not consist of individual, separate tracts of fibers. In comparison to these earlier CLSM fiber studies of the internal capsule, we generated PLI sections through the internal capsule in the horizontal and the sagittal plane (Figure 7). The heterogeneity in fiber orientation is clearly visible in the fiber orientation maps. However, single fibers running perpendicular to the majority of fibers in the anterior limb could not be detected with PLI (Figure 7E). Nevertheless, there is a good correlation between confocal images of fiber architecture and the larger-scale architectural pattern of PLI.

\section{EXTREME CAPSULE}

Recently, Saur et al. (2008) identified anatomical pathways connecting brain regions activated during two prototypical language tasks by combining fMRI with a DTI-based tractography. Tractography showed that language networks interact by distinct ventral (via the extreme capsule) and dorsal (via the superior longitudinal fasciculus) association tracts as well as commissural fibers (Saur et al., 2010). In order to validate the ventral stream anatomically, a human brain was macroscopically dissected using the method of Klingler (1935), i.e., the brain is frozen down to -5 to $-10^{\circ} \mathrm{C}$ and 


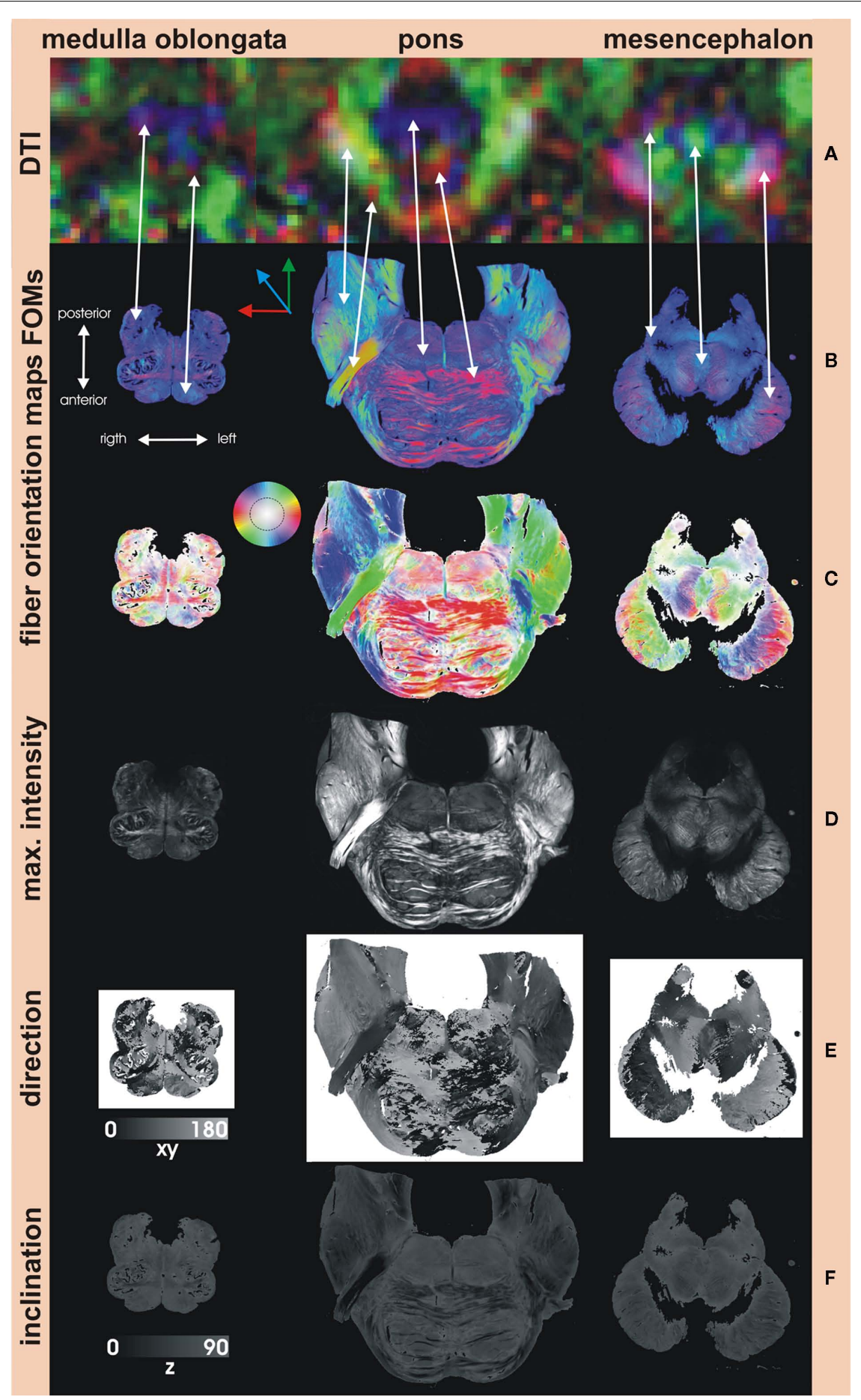

FIGURE 4 | Fiber Orientation Maps of the human brainstem.

(A) DTI color maps (1.5T MRI with a voxel resolution of

$2 \mathrm{~mm} \times 2 \mathrm{~mm} \times 2 \mathrm{~mm}$ ) of comparable sections through the brainstem are able to show the larger fiber tracts which at least are at the scale of one voxel. However, the resolution is too low to distinguish smaller structures as seen in the medulla oblongata. Corresponding fibers are marked by the arrows. The advantage of PLI is its greater resolution, which also allows for visualization of smaller fiber bundles. Fiber orientation maps (FOMs) can be visualized using a color scheme similar to the DTI slices with the absolute $\mathrm{X}$ component of the vector shown in red, the absolute of the $Y$ component in green, and the absolute of the $Z$ component in blue (B). Another color scheme (C) is more beneficial for PLI data and codes in-plane orientation in color and out-of-plane rotation in intensity (inset key). Maximum intensity maps (D) show the highest intensities of each polarization sequence and give relative good anatomical contrast similar to histological myelin stains. Angles of direction (in-plane) and angles of inclination (out-of-plane) generate direction (E) and inclination maps (F). Note that the angle of inclination can be estimated between $0^{\circ}$ and $90^{\circ}$ only, not distinguishable from angles between $0^{\circ}$ and $\left(-90^{\circ}\right.$ (inclination ambiguity). 


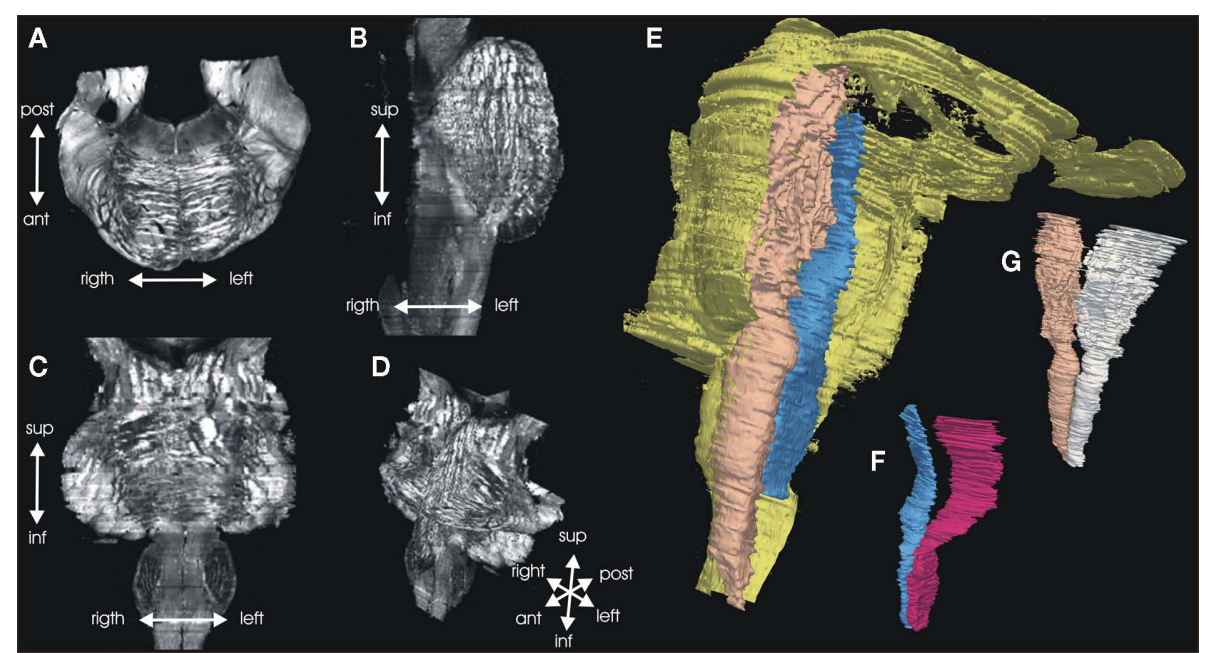

FIGURE 5 |Three dimension reconstruction of the brainstem. Serial FOMs are three-dimensionally reconstructed and a 3D data set is produced with a vector in each voxel representing the 3D fiber orientation. The example shows one original axial (A) section, and a calculated sagittal (B), and horizontal (C) section through the 3D data set (D) shown as maximum intensity data. Pyramidal tract and lemniscus medialis were manually segmented, based on the orientation data. Volume models of the whole brainstem [(E) yellow], pyramidal tracts $(\mathbf{G})$, and lemnisci mediales $(\mathbf{F})$ were computed.

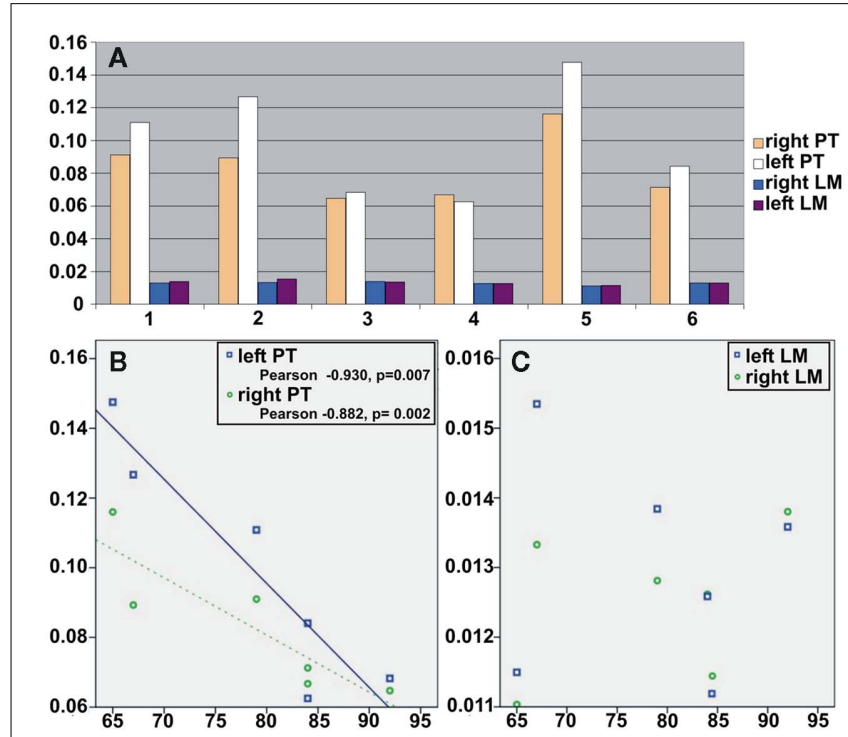

FIGURE 6 | Volumes of pyramidal tract and lemniscus medialis. The volumes of the pyramidal tracts and the lemnisci mediales were normalized to the whole white matter volume of the individual brainstem. The measurements of the analyzed six brainstems are shown in (A). The normalized volumes ( $y$-axis) of the pyramidal tracts revealed a negative correlation (B) to age ( $x$-axis), while the normalized volumes of the medial lemniscus were not correlated to age (C). Abbreviations: PT, pyramidal tract; LM, lemniscus medialis.

allowed to return to room temperature several times. The growing ice crystals lead to a slight separation of the nerve fibers from each other, allowing the fibers to be carefully dissected using fine forceps or blunt spatulas.

Macroscopically, distinct fiber systems could be differentiated which all channel in the depths of the limen insulae and proceed from the temporal to the frontal lobe: fibers from the extreme capsule to the frontal operculum, from the extreme capsule to the orbitofrontal cortex, and from the amygdala to the orbitofrontal cortex (the uncinate fasciculus; Figure 8). The uncinate fasciculus which is localized more deeply can be clearly distinguished from the other two fiber systems.

An additional brain (female, 85 years) was analyzed using PLI. For the investigation, a sagittal slab containing the limen insulae and the underlying white matter was dissected and serially sliced. Fiber orientation maps of these regions clearly reproduced the aforementioned fiber systems (Figure 8) as different fiber bundles. Therefore, we were able to anatomically reproduce the ventral stream system in the human brain using fiber dissection and PLI.

\section{DISCUSSION}

Studying the fiber anatomy of the human brainstem will not provide new insights into new fiber tracts or connectivity, but an analysis of these fiber tracts may be helpful for describing sidedependent asymmetries, and gender- or age-related differences. However, the low magnification of DTI (see also Figures 4A,B) in this region only allows evaluation of large fiber tracts, such as the pyramidal tract (Grässel et al., 2010).

Significantly, a negative relationship between age and the volumes of the pyramidal tracts (normalized to the whole white matter volume of the brainstem) was found in the current study. The result points to an age-related atrophy of the pyramidal tract system. In contrast, we found no age-related changes in the volume of the lemniscus medialis. Although, because of the small number of subjects analyzed in this study it is difficult to form reliable conclusions. Since it is obvious from Figures 6A,B that the three male subjects had larger pyramidal tract volumes and were younger than the female subjects, one might argue that these differences could be due to sex, age, both, or none of the above. Once again, the small sample size of three male and three female subjects, within a fairly 


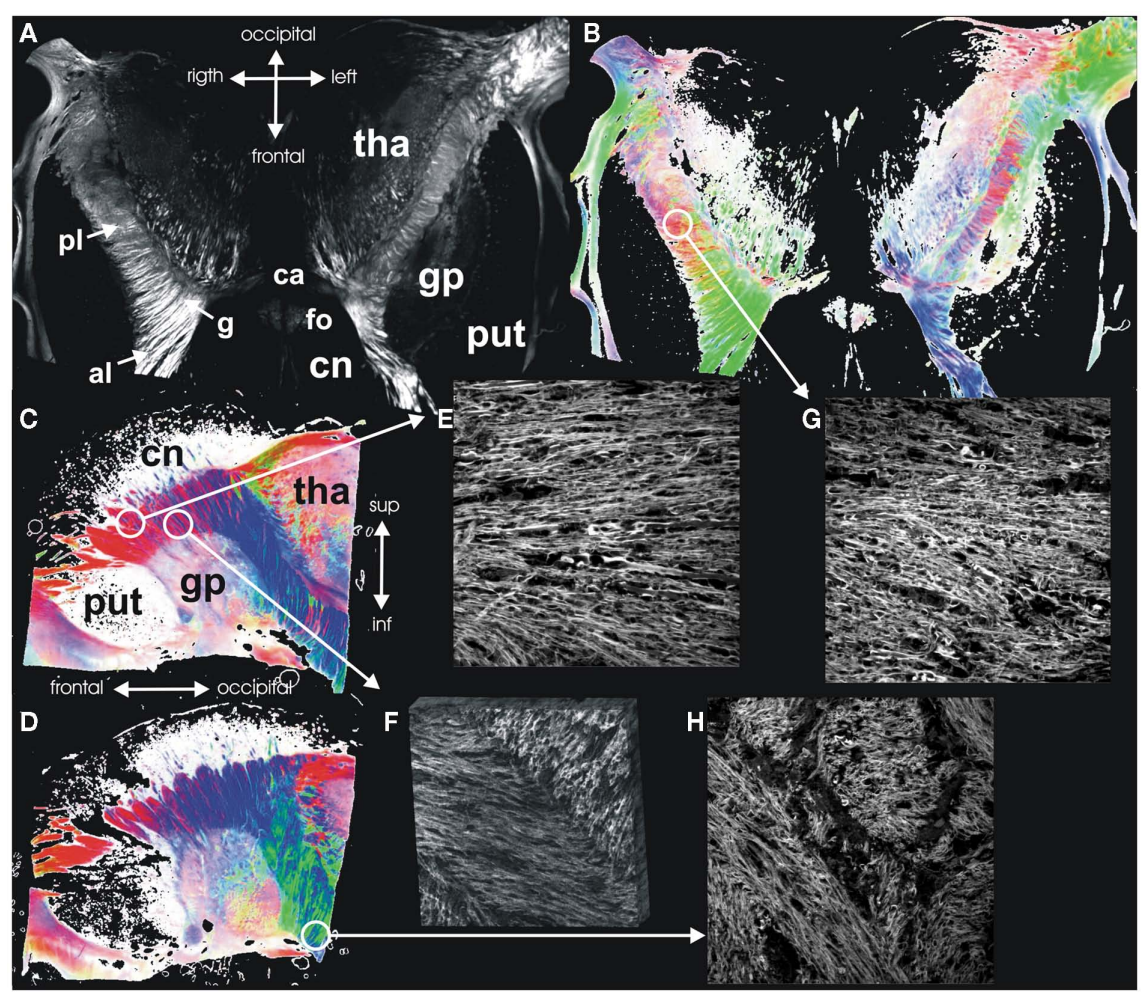

FIGURE 7 | Internal capsule. Maximum intensity map (A) and FOM (B) of a horizontal section through the internal capsule. (C,D) Show FOMs of sagittal sections through the internal capsule. Confocal images of different regions of the internal capsule demonstrate the fiber architecture at these points in high detail: (E) lateral anterior limb with parallel fibers and single nerve fiber crossing the internal capsule, (F) medial anterior limb with intermingling fiber bundles from the anterior thalamic radiation and the frontopontine fiber bundles (3D reconstruction), (G) posterior limb with intermingling fiber bundles from the pyramidal tract and superior thalamic radiation, $\mathbf{( H )}$ sublentiform part with larger intermingling compact bundles of fibers. Abbreviations: pl, posterior limb; g, genu; and al, anterior limb of the internal capsule; ca, anterior commissure; fo, fornix; gp, globus pallidus; pt, putamen; cn, caudate nucleus; tha, thalamus. limited age-range is not enough for a proper comparison, such as for laterality. Therefore, one can only form inspired hypotheses on this subject.

Currently, studies of age-related changes of distinct fiber tracts are sparse, although the numbers will certainly increase with a rise in the systematic use of MRI in this field (Wozniak and Lim, 2006). Typical DTI changes of white matter architecture over age are a decline of fractional anisotropy (FA) and an increase of diffusivity in normal healthy adults with an anterior-posterior gradient (Sullivan and Pfefferbaum, 2006; Yoon et al., 2008). In a voxel-based morphometric study of 465 healthy subjects, Good et al. (2001) found that the global white matter did not decline with age, but local areas of relative accelerated loss and preservation were seen primarily in the posterior limb of the internal capsule. Kawaguchi et al. (2010) recently found a decrease of FA in DTI datasets in the posterior limb of the internal capsule, which was interpreted as an atrophy of pyramidal tract fibers over age.

Most studies show a decline in FA and increase in diffusivity of white matter with age, which is typically interpreted as atrophy. However, to date these alterations can not be interpreted, since they could be due to changes in a number of factors including the number or density of myelinated axons, the spacing between axons, the branching patterns or crossing of axons, or the thickness of the axons and their myelin sheaths, to name only a few. PLI can provide information regarding fiber orientation only, although, data with respect to the number, density, and thickness is not available, while the grade of myelination of nerve fibers may be estimated from PLI data.

The internal capsule is an anatomical location where different projection fiber systems are closely packed in a small volume, in particular, the pyramidal tract, but also the thalamo-cortical, and the corticopontine fiber systems (Axer and Keyserlingk, 2000). Hence, this structure is the target of many studies using DTI (Kawaguchi et al., 2010). In this context, it is of significance to keep the complexity of this structure in mind in order to draw reliable conclusions from the results of DTI studies.

Connectivity and integrity of association pathways such as the cingulum and the extreme capsule have, for instance, a high impact on cognitive functioning (Rudrauf et al., 2008). The classical concept of language circuitry has been the connection between the temporal sensory region (Wernicke's area) and the frontal motor region (Broca's area) via the arcuate fasciculus (e.g., see Geschwind, 1970). However, modern concepts describe the classical dorsal pathway along the arcuate fasciculus/superior longitudinal fasciculus that is activated during repetition and a second ventral pathway via the extreme capsule which is activated 


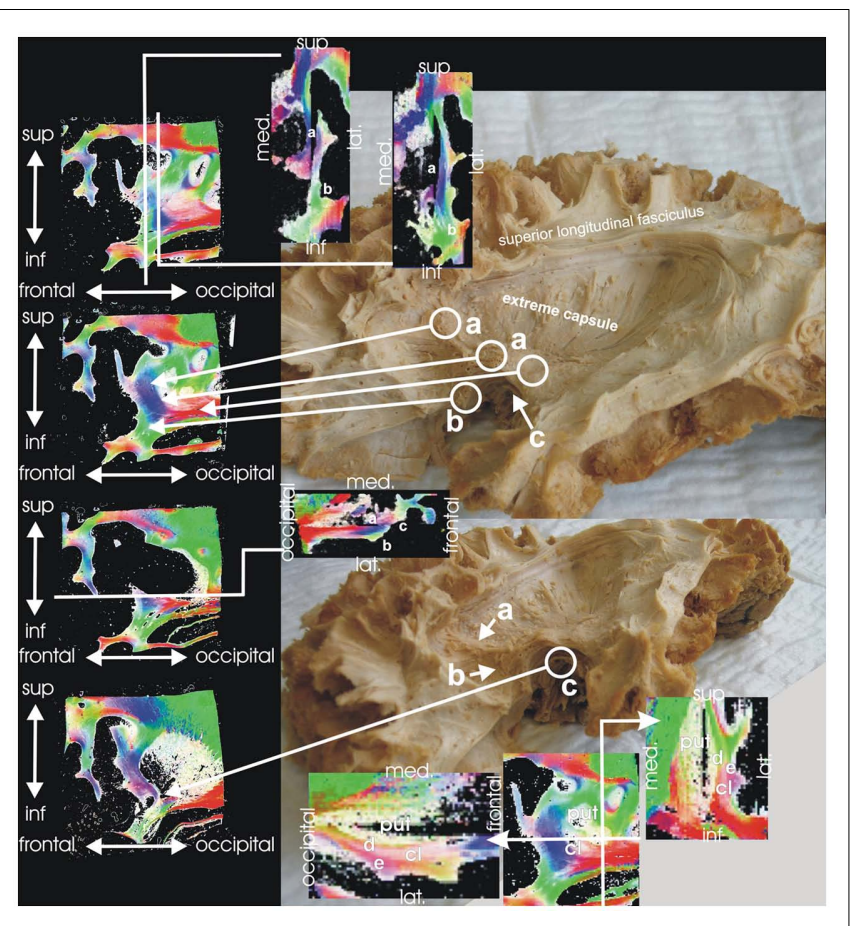

FIGURE 8 | Extreme capsule. Macroscopical fiber dissection and PLI of the white matter in the deep of the limen insulae show comparable results. Different nerve fibers can be distinguished running from the temporal to the frontal lobe: fibers from the extreme capsule to the frontal operculum (a), fibers from the extreme capsule to the orbitofrontal cortex (b), and fibers from the amygdala to the orbitofrontal cortex (c, the uncinate fasciculus). The uncinate fasciculus is localized more deeply than the other two fiber systems and can be clearly distinguished from these. The figures at the bottom show fiber orientation maps through putamen (put) and claustrum (cl), where external (d) and extreme capsule (e) can clearly be distinguished from each other.

during auditory comprehension (Saur et al., 2008). The extreme capsule seems to be part of a long association fiber pathway coursing between the inferior frontal region, the superior temporal gyrus, and the inferior parietal lobe (Makris and Pandya, 2009), which could be verified using DTI tractography of the human brain. In the monkey, these ventral and dorsal streams could also be demonstrated by means of tract tracing using radioactively labeled amino acids (Petrides and Pandya, 2009) as well as diffusion spectrum imaging (DSI; Schmahmann et al., 2007).

Anatomically, the white matter bridge between temporal and frontal lobes deep under the limen insulae is called the temporal stem (Kier et al., 2004). In this anatomical fiber channel, the extreme capsule fiber system can be clearly distinguished from the uncinate fasciculus, which is located deeper in the white matter. The uncinate fasciculus is a ventral limbic pathway (Schmahmann et al., 2007) connecting the rostral temporal region (including the amygdala) and the medial prefrontal cortex (especially the cingulate gyrus). The PLI results of the case study presented here could verify the anatomy of these fiber systems with respect to macroscopic dissection. Moreover, this topic is an example of changing concepts in the understanding of connectivity (of language circuits for instance), which was initially inspired by functional considerations.

The human cingulum bundle is another example of an association fiber pathway of the limbic system. Anatomically, the cingulum bundle is a collection of short and long association fibers which surround the corpus callosum (Nieuwenhuys et al., 1988). It is located above the corpus callosum and beneath the cingulate gyrus. The fiber bundle curves around the rostrum and genu of the corpus callosum spreading into the frontal lobe. Caudally, the bundle curves around the splenium of the corpus callosum and runs within the white matter of the parahippocampal gyrus.

In the monkey (Schmahmann et al., 2007), the fibers arise from the orbital surface (area 11 and 14) and areas 24 and 32 in the medial frontal cortex. Other fibers come from from SMA and areas 8,9 , and 46 in the dorsolateral prefrontal cortex. In addition, caudal connections also exist with the inferior parietal lobule and parahippocampal regions.

The cingulate gyrus may be subdivided into the anterior, mid, posterior, and retrosplenial cortices (ACC, MCC, PCC, and RSC; Vogt, 2005; Palomero-Gallagher et al., 2009). The midcingulate cortex (MCC) receives more input from the inferior parietal cortex and less from the amygdala compared to the perigenual cingulate cortex (Vogt and Pandya, 1987; Vogt et al., 2003). MCC contains the cingulate motor area (CMA), which has similar connections like the premotor areas with reciprocal connections with primary motor cortex and supplementary motor area (Devinsky et al., 1995). The anterior cingulate gyrus is believed to be primarily involved in executive functions especially of emotion, visceromotor and skeletomotor control, vocalization, and pain.

The anterior executive anterior cingular cortex (Devinsky et al., 1995; Allman et al., 2001) may further be subdivided into a ventral affect-dominated division, mainly connected to the amygdala and the periaqueductal gray as well as a dorsal cognition-dominated division characterized by its contribution to nociception and skeletomotor control. This region has projections to all parts of the striatum.

The PLI data regarding fiber architecture of the anterior cingulum bundle suggests a homologous parcellation. The posterior part of the supracallosal cingulum bundle receives various inputs from the adjacent white matter that apparently comes from the motor- and premotor areas and parietal cortex. In contrast, the subgenual part shows manifold connections to the orbitofrontal areas as well as with fibers directed to the amygdala. In this region, the cingulum bundle has a high variability (Bürgel et al., 2006). Our data demonstrate that these fibers are diffusely intermingled with fibers of the medial forebrain bundle and laterally with the forceps minor of the corpus callosum.

Generally, PLI is a method which supplies information relating to fiber orientation. Therefore, it provides similar information as attained from diffusion MRI, although both methods estimate fiber orientation based on different physical principles (diffusion vs. optical birefringence). The advantage of PLI is that it can provide higher resolution in comparison to diffusion MRI. For instance, a problem with diffusion-based methods is that those cannot differentiate between external and extreme capsules. PLI is able to resolve these fibers (see Figure 8). The resolution as used in the studies presented here was $64 \mu \mathrm{m} \times 64 \mu \mathrm{m} \times 100 \mu \mathrm{m}$ 
per voxel. However, resolution of the method can be enhanced by using a microscope for imaging (Larsen et al., 2007). Nevertheless, to maximize resolution, it is necessary to use sections that are as thin as possible, so that section thickness may the major limiting factor of resolution.

Inclination ambiguity is a second limiting aspect of PLI, as fiber inclination (out-of-plane orientation) can be estimated only between $0^{\circ}$ and $90^{\circ}$ (e.g., an inclination of $45^{\circ}$ and $-45^{\circ}$ will give the same signal and cannot be distinguished from each other). However, physical improvements of the imaging system may overcome this problem in the near future. The progress in computational methods allows for elastic 3D reconstruction and finally for $3 \mathrm{D}$ tractography in PLI datasets (Axer et al., 2011). Although, PLI is a method which is actually still in a state of development, histological processing of the specimens is time-consuming and elaborate and as such the use of the method will be restricted to a limited number of laboratories and to a limited number of samples.

On a clinical MRI scanner, the order of a voxel size is in the $\mathrm{mm}$ range. Thus, one voxel can contain hundreds of thousand axons with a total length of $1 \mathrm{~km}$ (Alexander and Seunarine, 2011). These axons will probably have different orientations in all possible variations. Very problematic configurations involve crossing fibers or kissing fibers. Further, the classic diffusion tensor only represents one major fiber orientation. In contrast, fiber orientation distribution function (fODF) quantifies the fraction of fibers in the voxel pointing in each direction. This, therefore, represents a more differentiated estimate of the complex voxel composition. In diffusion MRI, estimations of the fODF may be achieved by the use of DSI on high-end magnets or single shell high angular resolution diffusion imaging (HARDI) without major hardware requirements or others (Hagmann et al., 2010). These methods have been specifically developed to solve the crossing fiber problem in MR. It can be used to perform fiber tracking in a much more robust and reliable manner (Tournier, 2011).

Polarized light imaging provides information regarding a single direction in each voxel. The difference to diffusion MRI, however, is the smaller voxel size, so that about 1000 PLI voxels are contained in one voxel of diffusion MRI. Therefore, the higher magnification of PLI allows for the imaging of smaller bundles of fibers and, therefore, will reduce problems of crossing and kissing fibers since inhomogeneous composition of the voxels is consequently reduced. However, PLI does not provide information about fiber number or fiber density, while the degree of myelination may be a parameter which can be estimated from the measurements. PLI as an estimate of fiber orientation is, therefore, very similar to DTI, which represents a dataset of principal diffusion directions.

MR-based methods, such as DTI, DSI, and also fMRI together with other examples, have the clear advantage that they allow for investigations of the living human brain over time in healthy or diseased conditions. However, an established gold standard for reference purposes is not yet available (Hagmann et al., 2010). To perform a multi-methodological validation, the combination of different anatomical methods has to be undertaken on the same, or at least on comparable objects. Postmortem studies are indispensable for this purpose. Several anatomical methods exist to study fiber architecture (Axer, 2011), e.g., macroscopic dissection or confocal microscopy as seen in our examples. Recently, a very promising tool to generate a highly resolved 3D data set of a whole Golgi-stained mouse brain has been published (Li et al., 2010). This system is able to generate comprehensive $3 \mathrm{D}$ data of a complete mouse brain, but is to date not applicable to entire human brains.

Each method only shows a selective view of the object, for example connectivity, number of fibers, myelo- and cytoarchitectural histology, or fiber orientation. The analysis of the extreme capsule system as presented here shows that the combination of different methods (e.g., DTI tractography and fMRI (Saur et al., 2010)) generates new concepts, which, in turn, may be evaluated using other neuroanatomic methods. Moreover, it represents a paradigm of the interpretation of the functional meaning of fiber tracts and connectivity, but has to be proven using different anatomical methodological approaches - since at the end, the basis of these functions is the structure of white and gray matter.

\section{CONCLUSION}

In the recent past, the discussion regarding the exploration of the human connectome as a compilation of the overall connectivity in human brain has gained increasing attention (Sporns, 2011). To date, several methods for connectivity mapping are available ranging from macroscopic to molecular scales. However, it is difficult to integrate all these multiple scaled data into one concept. It is not yet clear what the optimal scale is for efficient characterization of brain connectivity (Hagmann et al., 2010). Perhaps a courser to finer approach may be a solution achieved by using MR methods to generate "larger-scale" hypotheses which could create specified studies using "micro-scale" methods (see also DeFelipe, 2010).

However, connectivity is not only a matter of scale, but also a matter of modality and function. This is largely influenced by the scientific method which is used and the conditions under which scientific interest the study has been performed, i.e., the hypothesis that has to be proven. Challenging targets in connectivity may be variability, age-, and gender-dependent differences, asymmetry, development, degeneration, plasticity, and disease-specific changes of nerve fiber architecture.

Polarized light imaging - in this context - is a method, which can estimate fiber orientation in gross histological sections of the human brain in great detail. Although, it provides a dataset of principal fiber directions, it has the appealing advantage of a much higher resolution than MR-based methods. Therefore, PLI can generate high-resolved 3D datasets of fiber orientation of the human brain and it has, therefore, a high comparability to diffusion MR. To obtain additional information regarding axon structure and density, PLI can also be combined with classical histological stains. It brings the directional aspects of diffusion MRI into the range of histology and may represent a promising tool to close the gap between larger-scale diffusion orientation and microstructural histological analysis of connectivity.

\section{ACKNOWLEDGMENTS}

This work was partly funded by the German Research Counsil (DFG, Deutsche Forschungsgemeinschaft; grant no. Ax 20/3-1) and the German Federal Ministry of Education and Research (BMBF, grant no. 01GW0740). We thank Nasim Kroegel for her help in language editing. 


\section{REFERENCES}

Aboitiz, F., Scheibel, A. B., Fisher, R. S., and Zaidel, E. (1992). Fiber composition of the human corpus callosum. Brain Res. 598, 143-153.

Alexander, D. C., and Seunarine, K. K. (2011). "Mathematics of crossing fibers," in Diffusion MRI: Theory, Methods, and Applications, ed. D. K. Jones (New York: Oxford University Press), 451-464.

Allman, J. M., Hakeem, A., Erwin, J. M., Nimchinsky, E., and Hof, P. (2001). The anterior cingulate cortex. The evolution of an interface between emotion and cognition. Ann. N. Y. Acad. Sci. 935, 107-117.

Axer, H. (2011). "Invasive methods for tracing white matter architecture," in Diffusion MRI: Theory, Methods, and Applications, ed. D. K. Jones (New York: Oxford University Press), 31-42.

Axer, H., Axer, M., Krings, T., and Keyserlingk, D. G. (2001). Quantitative estimation of 3-D fiber course in gross histological sections of the human brain using polarized light. J. Neurosci. Methods 105, 121-131.

Axer, H., Berks, G., and Keyserlingk, D. G. (2000). Visualization of nerve fiber orientation in gross histological sections of the human brain. Microsc. Res. Tech. 51, 481-492.

Axer, H., Grässel, D., Steinhauer, M., Stöhr, P., John, A., Coenen, V. A., Jansen, R. H., and Keyserlingk, D. G. (2002a). Microwave dielectric measurements and tissue characteristics of the human brain: potential in localizing intracranial tissues. Phys. Med. Biol. 47, 1793-1803.

Axer, H., Leunert, M., Mürköster, M., Grässel, D., Larsen, L., Griffin, L. D., and Keyserlingk, D. G. (2002b). A 3D fiber model of the human brainstem. Comput. Med. Imaging Graph 26, 439-444.

Axer, H., and Keyserlingk, D. G. (2000). Mapping of fiber orientation in human internal capsule by means of polarized light and confocal scanning laser microscopy. J. Neurosci. Methods 94, 165-175.

Axer, H., Lippitz, B. E., and Keyserlingk, D. G. (1999a). Morphological asymmetry in anterior limb of human internal capsule revealed by confocal laser and polarized light microscopy. Psychiatry Res. 91, 141-154.

Axer, H., Stegelmeyer, J., and Keyserlingk, D. G. (1999b). Comparison of tissue impedance measurements with nerve fiber architecture in human telencephalon: value in identification of intact subcortical structures. J. Neurosurg. 90, 902-909.

Axer, M., Amunts, K., Grässel, D., Palm, C., Dammers, J., Axer, H., Pietrzyk,
U., and Zilles, K. (2011). A novel approach to the human connectome: ultra-high resolution mapping of fiber tracts in the brain. Neuroimage 54, 1091-1101.

Briggman, K. L., and Denk, W. (2006). Towards neural circuit reconstruction with volume electron microscopy techniques. Curr. Opin. Neurobiol. 16, 562-570.

Brodmann, K. (1903). Bemerkungen zur Untersuchung des Nervensystems im polarisierten Lichte. J. Psychol. Neurol. 2, 211-213.

Brosseau, C. (1998). Polarized Light A Statistical Optics Approach. New York: John Wiley \& Sons.

Bürgel, U., Amunts, K., Hoemke, L., Mohlberg, H., Gilsbach, J. M., and Zilles, K. (2006). White matter fiber tracts of the human brain: threedimensional mapping at microscopic resolution, topography and intersubject variability. Neuroimage 29, 1092-1105.

Catani, M., Howard, R. J., Pajevic, S., and Jones, D. K. (2002). Virtual in vivo interactive dissection of white matter fasciculi in the human brain. Neuroimage 17, 77-94.

DeFelipe, J. (2010). From the connectome to the synaptome: an epic love story. Science 330, 1198-1201.

Devinsky, O., Morrell, M. J., and Vogt, B. A. (1995). Contributions of anterior cingulate cortex to behaviour. Brain 118, 279-306.

Finsterbusch, J. (2009). High-resolution diffusion tensor imaging with inner field-of-view EPI. J. Magn. Reson. Imaging 29, 987-993.

Gering, D. T., Nabavi, A., Kikinis, R., Hata, N., O’Donnell, L. J., Grimson, W. E., Jolesz, F. A., Black, P. M., and Wells, W. M. (2001). An integrated visualization system for surgical planning and guidance using image fusion and an open MR. J. Magn. Reson. Imaging 13, 967-975.

Geschwind, N. (1970). The organization of language and the brain. Science 170, 940-944.

Good, C. D., Johnsrude, I. S., Ashburner, J., Henson, R. N., Friston, K. J., and Frackowiak, R. S. (2001). A voxelbased morphometric study of ageing in 465 normal adult human brains. Neuroimage 14, 21-36.

Göthlin, G. (1913). Die doppelbrechenden Eigenschaften des Nervengewebes. Uppsala: Friedländer \& Sohn.

Graf von Keyserlingk, D., and Schramm, U. (1984). Diameter of axons and thickness of myelin sheaths of the pyramidal tract fibres in the adult human medullary pyramid. Anat. Anz. 157, 97-111.

Grässel, D., Ringer, T. M., Fitzek, C., Fitzek, S., Kohl, M., Kaiser, W. A., Witte, O. W., and Axer, H.
(2010). Wallerian degeneration of pyramidal tract after paramedian pons infarct. Cerebrovasc. Dis. 30 380-388.

Hagmann, P., Cammoun, L., Gigandet, X., Gerhard, S., Grant, P. E., Wedeen, V., Meuli, R., Thiran, J.-P., Honey, C. J., and Sporns, O. (2010). MR connectomics: principles and challenges. J. Neurosci. Methods 194 , 34-45.

Jones, D. K. (2011). Diffusion MRI: Theory, Methods, and Applications, 1st Edn. New York: Oxford University Press.

Jones, R. C. (1941). A new calculus for the treatment of optical systems. J. Opt. Soc. Am. 31, 488-493.

Kawaguchi, H., Obata, T., Ota, M. Akine, Y., Ito, H., Ikehira, H., Kanno, I., and Suhara, T. (2010). Regional heterogeneity and agerelated change in sub-regions of internal capsule evaluated by diffusion tensor imaging. Brain Res. 1354 30-39.

Kier, E. L., Staib, L. H., Davis, L. M., and Bronen, R. A. (2004). MR imaging of the temporal stem: anatomic dissection tractography of the uncinate fasciculus, inferior occipitofrontal fasciculus, and Meyer's loop of the optic radiation. AJNR Am. J. Neuroradiol. 25, 677-691.

Klingler, J. (1935). Erleichterung der makroskopischen Präparation des Gehirns durch den Gefrierprozeß. Schweiz. Arch. Neurol. 36, 247-256.

Kretschmann, H. J. (1967). On the demonstration of myelinated nerve fibers by polarized light without extinction effects. J. Hirnforsch. 9, 571-575.

Larsen, L., Griffin, L. D., Grässel, D. Witte, O. W., and Axer, H. (2007). Polarized light imaging of white matter architecture. Microsc. Res. Tech. 70, 851-863.

Li, A., Gong, H., Zhang, B., Wang, Q., Yan, C., Wu, J., Liu, Q., Zeng, S., and Luo, Q. (2010). Microoptical sectioning tomography to obtain a high-resolution atlas of the mouse brain. Science 330, 1404-1408.

Ludwig, E., and Klingler, L. (1956). Atlas cerebri humani. Basel: Karger.

Makris, N., and Pandya, D. N. (2009). The extreme capsule in humans and rethinking of the language circuitry. Brain Struct. Funct. 213, 343-358.

Martenson, R. E. (1992). Myelin: Biology and Chemistry. Boca Raton: CRC Press.

Miklossy, J., and Van der Loos, H. (1991). The long-distance effects of brain lesions: visualization of myelinated pathways in the human brain using polarizing and fluorescence microscopy. J. Neuropathol. Exp. Neurol. 50, 1-15.

Nieuwenhuys, R., Voogd, J., and Van Huijzen, C. (1988). The Human Central Nervous System. Berlin: Springer.

Palomero-Gallagher, N., Vogt, B. A., Schleicher, A., Mayberg, H. S., and Zilles, K. (2009). Receptor architecture of human cingulate cortex: evaluation of the four-region neurobiological model. Hum. Brain Mapp. 30, 2336-2355.

Petrides, M., and Pandya, D. N. (2009). Distinct parietal and temporal pathways to the homologues of Broca's area in the monkey. PLoS Biol. 7, e1000170. doi: 10.1371/journal.pbio. 1000170

Rudrauf, D., Mehta, S., and Grabowski, T. J. (2008). Disconnection's renaissance takes shape: formal incorporation in group-level lesion studies. Cortex 44, 1084-1096.

Saur, D., Kreher, B. W., Schnell, S., Kümmerer, D., Kellmeyer, P., Vry, M.-S., Umarova, R. Musso, M., Glauche, V., Abel, S., Huber, W., Rijntjes, M., Hennig, J., and Weiller, C. (2008). Ventral and dorsal pathways for language. Proc. Natl. Acad. Sci. U.S.A. 105, 18035-18040.

Saur, D., Schelter, B., Schnell, S., Kratochvil, D., Küpper, H., Kellmeyer, P., Kümmerer, D., Klöppel, S., Glauche, V., Lange, R., Mader, W., Feess, D., Timmer, J., and Weiller, C. (2010). Combining functional and anatomical connectivity reveals brain networks for auditory language comprehension. Neuroimage 49, 3187-3197.

Scheuner, G., and Hutschenreuter, J. (1972). Polarisationsmikroskopie in der Histophysik. Leipzig: VEB Georg Thieme.

Schmahmann, J. D., Pandya, D. N., Wang, R., Dai, G., D’Arceuil, H. E., de Crespigny, A. J., and Wedeen, V. J. (2007). Association fibre pathways of the brain: parallel observations from diffusion spectrum imaging and autoradiography. Brain 130, 630-653.

Schmidt, W. (1924). Die Bausteine des Tierkörpers im polarisierten Lichte. Bonn: Cohen.

Schmitt, F. O., and Bear, R. S. (1935). The optical properties of vertebrate nerve axons are related to fiber size. J. Cell. Comp. Physiol. 9, 261-273.

Schüz, A., and Preißl, H. (1996). Basic connectivity of the cerebral cortex and some considerations on the corpus callosum. Neurosci. Biobehav. Rev. 20, 567-570.

Sporns, O. (2011). The human connectome: a complex network. Ann. N. Y. Acad. Sci. 1224, 109-125. 
Sporns, O., Tononi, G., and Kötter, R. (2005). The human connectome: a structural description of the human brain. PLoS Comput. Biol. 1, e42. doi: 10.1371/journal.pcbi.0010042

Sullivan, E. V., and Pfefferbaum, A. (2006). Diffusion tensor imaging and aging. Neurosci. Biobehav. Rev. 30, 749-761.

Tournier, J.-D. (2011). “The biophysics of crossing fibers," in Diffusion MRI: Theory, Methods, and Applications, ed. D. K. Jones (New York: Oxford University Press), 465-481.

Vidal, B., Silveira Mello, M. L., CaseiroFilho, A. C., and Godo, C. (1979). Anisotropic properties of the myelin sheath. Acta Histochem. 66, 32-39.

Vogt, B. A. (2005). Pain and emotion interactions in subregions of the cingulate gyrus. Nat. Rev. Neurosci. 6, 533-544.

Vogt, B. A., Berger, G. R., and Derbyshire, S. W. G. (2003). Structural and functional dichotomy of human midcingulate cortex. Eur. J. Neurosci. 18, 3134-3144.

Vogt, B. A., and Pandya, D. N. (1987). Cingulate cortex of the rhesus monkey: II. Cortical afferents. J. Comp. Neurol. 262, 271-289.

Wolman, M. (1970). On the use of polarized light in pathology. Pathol. Annu. 5, 381-416.

Wolman, M. (1975). Polarized light microscopy as a tool of diagnostic pathology. J. Histochem. Cytochem. 23, 21-50.

Wozniak, J. R., and Lim, K. O. (2006). Advances in white matter imaging: a review of in vivo magnetic resonance methodologies and their applicability to the study of development and aging. Neurosci. Biobehav. Rev. 30, 762-774.

Wright, S. J., Centonze, V. E., Stricker, S. A., DeVries, P. J., Paddock, S. W., and Schatten, G. (1993). Introduction to confocal microscopy and threedimensional reconstruction. Methods Cell Biol. 38, 1-45.

Yoon, B., Shim, Y.-S., Lee, K.-S., Shon, Y.-M., and Yang, D.-W. (2008). Region-specific changes of cerebral white matter during normal aging: a diffusion-tensor analysis. Arch. Gerontol. Geriatr. 47, 129-138.

Zhang, J., Jones, M. V., McMahon, M. T. Mori, S., and Calabresi, P. A. (2011) In vivo and ex vivo diffusion tensor imaging of cuprizone-induced demyelination in the mouse corpus callosum. Magn. Reson. Med. doi: 10.1002/mrm.23032 [Epub ahead of print].

Conflict of Interest Statement: The authors declare that the research was conducted in the absence of any commercial or financial relationships that could be construed as a potential conflict of interest.

Received: 01 March 2011; accepted: 25 October 2011; published online: 11 November 2011.

Citation: Axer $H$, Beck $S$, Axer $M$, Schuchardt F, Heepe J, Flücken $A$, Axer $M$, Prescher $A$ and Witte OW (2011) Microstructural analysis of human white matter architecture using polarized light imaging: views from neuroanatomy. Front. Neuroinform. 5:28 doi: 10.3389/fninf.2011.00028

Copyright (C) 2011 Axer, Beck, Axer, Schuchardt, Heepe, Flücken, Axer, Prescher and Witte. This is an openaccess article subject to a non-exclusive license between the authors and Frontiers Media SA, which permits use, distribution and reproduction in other forums, provided the original authors and source are credited and other Frontiers conditions are complied with. 